\title{
Targeted Temperature Management Following Cardiac Arrest: In-Hospital Trends in Utilization
}

\author{
Saiprasad Narsingam ${ }^{1 *}$, Benjamin S Abella ${ }^{2}$, Anne V Grossestreuer ${ }^{2}$ and Paul S Chan ${ }^{1,3}$ \\ ${ }^{1}$ University of Missouri-Kansas City, Kansas City, USA \\ ${ }^{2}$ Center for Resuscitation Science, University of Pennsylvania, Philadelphia, USA \\ ${ }^{3}$ Saint Luke's Mid America Heart Institute, Kansas City, USA
}

*Corresponding author: Dr. Saiprasad Narsingam, University of Missouri-Kansas City School of Medicine, 2411 Holmes Street, Kansas City, MO 64108, USA, E-mail: narsingam.sp@gmail.com

\begin{abstract}
Background: Targeted Temperature Management (TTM) improves outcomes in patients with Out-of-Hospital Cardiac Arrest (OHCA) due to Shockable rhythms. The frequency with which TTM is used for Non-Shockable cardiac arrest rhythms and for In-Hospital Cardiac Arrest (IHCA) is unknown.

Methods: Within the prospective multicenter Penn Alliance for Therapeutic Hypothermia registry we examined the proportion of patients treated with TTM for OHCA vs. IHCA, and for Shockable vs. Non-Shockable cardiac arrest rhythms.
\end{abstract}

Results: Of 976 patients treated with TTM, 785 (80.4\%) were for OHCA, with a trend ( $P$ for trend of 0.05 ) for a higher proportion of cardiac arrests due to OHCA over time. Most (523 [56.8\%]) of those treated with TTM had Non-Shockable cardiac arrest rhythms. Notably, TTM was initiated for OHCA due to a Shockable cardiac arrest rhythm in only 349 $(37.9 \%)$ patients, although the proportion has increased over time $(30.6 \%$ in $2010 ; 45.2 \%$ in 2014 ; $P$ for trend of 0.04).

Conclusion: In real-world practice, only 2 in 5 cardiac arrest patients treated with TTM are for OHCA due to a Shockable rhythm. Given uncertain observational study results for TTM for IHCA and Non-Shockable cardiac arrest rhythms, there remains a need for prospective trials of TTM for these other treatment indications.

\section{Keywords}

Hypothermia, Induced, Out-of-Hospital cardiac arrest, Ventricular fibrillation, Tachycardia, Ventricular, Heart arrest, Trends, Cardiopulmonary resuscitation

\section{Introduction}

Cardiac arrest occurs commonly, with over 325,000 cases of Out-of-Hospital Cardiac Arrest (OHCA) and 200,000 cases of In-Hospital Cardiac Arrests (IHCA) occurring annually in the United States [1]. Given low survival rates for both OHCA and IHCA, strategies such as post-arrest application of TTM have been developed to improve survival. TTM decreases cerebral oxygen demand and free radical formation and is thought to mitigate cerebral reperfusion injury after return of spontaneous circulation is achieved post cardiac arrest $[2,3]$. The exact mechanism by which TTM is neuro-protectiveis believed to be through avoidance of cerebral perfusion injury. This concept was successfully tested in humans with OHCA due to Shockable rhythms such as Ventricular Fibrillation and Ventricular Tachycardia (VF/VT), wherein patients randomized to TTM were shown to have both higher rates of survival and favorable neurological outcome in two multicenter clinical trials $[4,5]$.

Since the publication of these two landmark trials, TTM has become a standard treatment for cardiac arrest at many hospitals. However, its efficacy for Non-Shockable cardiac arrest rhythms, such as asystole and Pulseless Electrical Activity (PEA), is unclear. Some observational studies have suggested that TTM treatment in OHCA patients with Non-Shockable cardiac arrest rhythms may be ineffectual, and a recent meta-analysis reported, at best, a very small benefit but noted that almost all of the studies were of low quality evidence [6].

Citation: Narsingam S, Abella BS, Grossestreuer AV, Chan PS (2017) Targeted Temperature Management Following Cardiac Arrest: In-Hospital Trends in Utilization. Int J Crit Care Emerg Med 3:026. doi. org/10.23937/2474-3674/1510026

Received: June 10, 2016: Accepted: May 13, 2017: Published: May 15, 2017

Copyright: (c) 2017 Narsingam S, et al. This is an open-access article distributed under the terms of the Creative Commons Attribution License, which permits unrestricted use, distribution, and reproduction in any medium, provided the original author and source are credited. 
Similarly, whether TTM treatment improves survival for patients with IHCA-in which response times, comorbidity burden, and cardiac arrest etiology differ markedly from OHCA-has not been established, and threeobservational studies have not shown a survival benefit with TTM treatment [7-9]. Despite this, current guidelines continue to recommend TTM in comatose cardiac arrest survivors even with Non-Shockable rhythms and IHCA based on expert consensus alone [10,11].

Given the lack of efficacy studies for TTM treatment beyond patients with OHCA due to VF/VT, it would be important to understand contemporary patterns of its use for cardiac arrest in routine practice. If the majority of treated patients have IHCA or a Non-Shockable cardiac arrest rhythm, it may suggest a need for additional clinical trials to examine treatment efficacy in these settings, especially given existing results from observational studies. Accordingly, we examined contemporary TTM treatment patterns for cardiac arrest (OHCA vs. IHCA; Shockable vs. Non-Shockable rhythms) and whether this has changed over time.

\section{Methods}

We used data from the Penn Alliance for Therapeutic Hypothermia (PATH) registry, a multicenter internet-based clinical registry that was developed to collect information for research on TTM treatment for cardiac arrest [12,13]. Established in 2010 by investigators at the University of Pennsylvania, participating U.S. hospitals collect data on patients resuscitated from IHCA and OHCA using Utstein style definitions, with a focus on post-resuscitation care, including information on TTM use [14]. Member institutions submit data on 30 re- quired elements for each cardiac arrest patient as well as an additional 100 optional data elements. For each patient with cardiac arrest, PATH collects a variety of data on pre-, intra- and post-cardiac arrest factors. For the purpose of this paper, we were primarily interested in whether a patient received TTM treatment for IHCA vs. OHCA and for which type of underlying cardiac arrest rhythm from January $1^{\text {st }}, 2010$ through November $30^{\text {th }}$, 2014.

\section{Statistical analysis}

Baseline characteristics of patients treated and not treated with TTM were compared using descriptive statistics, including chi-square tests for categorical variables. Similarly, baseline characteristics of TTM-treated patients with a Shockable (VF and pulseless VT) vs. Non-Shockable (asystole and PEA) cardiac arrest rhythm were compared.

To examine trends in the proportion of patients treated with TTM with an OHCA vs. an IHCA, we first calculated the proportion of cases for each treated with TTM for each 3-month calendar year quarter over the 5 -year study period. We then constructed linear regression models to assess whether the proportion of TTM-treated patients with an OHCA has increased, decreased, or remained unchanged over the 20 calendar year quarters using linear tests of trends. Similarly, to examine trends in the proportion of patients treated with TTM with a Shockable vs. Non-Shockable cardiac arrest rhythm, we calculated the proportion of TTM-treated patients in each group by calendar year quarters and assessed whether the proportion of TTM-treated patients with a Shockable cardiac arrest rhythm has increased,

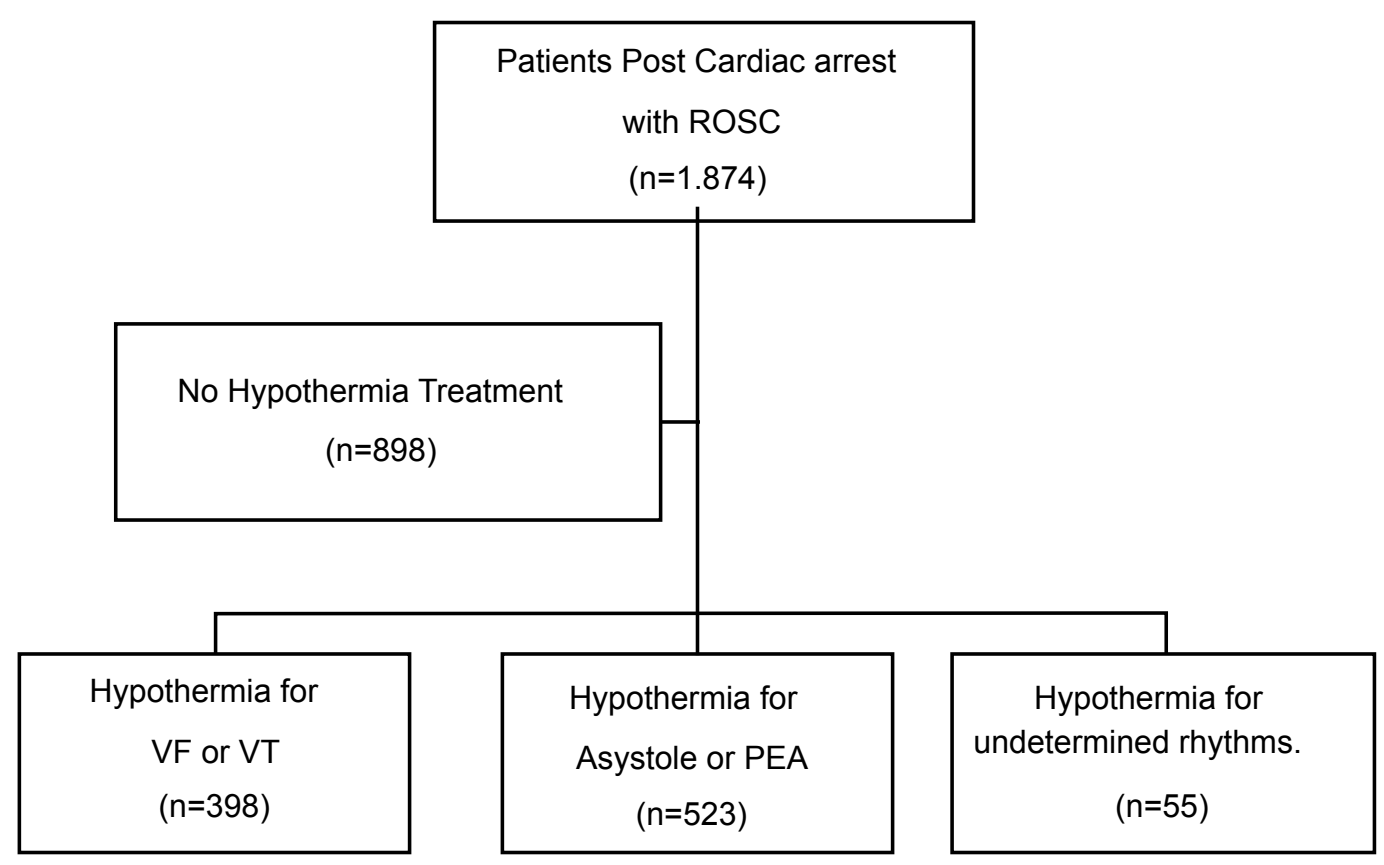

Figure 1: Definition of the study cohort.

Abbreviation: PEA: Pulseless Electrical Activity; ROSC: Return of Spontaneous Circulation; VF: Ventricular Fibrillation; VT: Pulseless Ventricular Tachycardia. 
decreased, or remained unchanged over time using linear regression models. Finally, we evaluated whether the proportion of TTM-treated patients with an OHCA due to a Shockable cardiac arrest rhythm-the indication for which TTM has been shown to be efficacious in randomized trials-has increased over time using similar analyses.

All analyses were performed using SAS (Version 9.3, Cary, N.C.) and evaluated at a 2-sided significance level of 0.05. Requirement for informed consent was waived by the institutional review board at Saint Luke's Hospital, as the study used de-identified patient data.

\section{Results}

Between January 2010 and November 2014, a total of 1,874 patients who had a cardiac arrest were enrolled in the PATH registry irrespective of whether TTM was instituted. Of these, 976 patients (52.1\%) from 26 hospitals were treated with TTM as part of post-cardiac arrest care (Figure 1). All patients were treated with a target temperature between $32{ }^{\circ} \mathrm{C}-34{ }^{\circ} \mathrm{C}$. Overall, patients of younger age, male sex, with an Out-of-Hospital cardiac arrest, a cardiac arrest etiology, and an unwitnessed arrest were more likely to be treated with TTM (Table 1). Moreover, TTM patients were more likely to

Table 1: Baseline characteristics of patients treated and not treated with TTM within the path registry.

\begin{tabular}{|c|c|c|c|}
\hline & TTM & No TTM & \\
\hline & $(n=976)$ & $(n=898)$ & $P$ value \\
\hline Male Sex (\%) & $603(61.8)$ & $507(56.5)$ & 0.01 \\
\hline Median Age (IQR), Years & $62(51-73)$ & $68(56-78)$ & \\
\hline Initial Cardiac Arrest Rhythm (\%) & & & $<0.001$ \\
\hline Asystole (Non-shockable) & $207(22.5)$ & $190(22.8)$ & \\
\hline PEA (Non-shockable) & $316(34.3)$ & $415(49.8)$ & \\
\hline VF/VT (Shockable) & $398(43.2)$ & $229(27.5)$ & \\
\hline Unknown & 55 & 64 & \\
\hline Witnessed (\%) & $699(71.6)$ & $745(83.0)$ & $<0.001$ \\
\hline Cardiac Etiology of Arrest (\%) & $616(63.1)$ & $504(56.1)$ & 0.001 \\
\hline Location of Cardiac Arrest (\%) & & & $<0.001$ \\
\hline In-Hospital & $191(19.6)$ & $665(74.1)$ & \\
\hline Out-of-Hospital & $785(80.4)$ & $233(25.9)$ & \\
\hline
\end{tabular}

Abbreviations: IQR: Inter-Quartile Range; PEA: Pulseless Electrical Activity; VF: Ventricular Fibrillation; VT: Pulseless Ventricular Tachycardia.

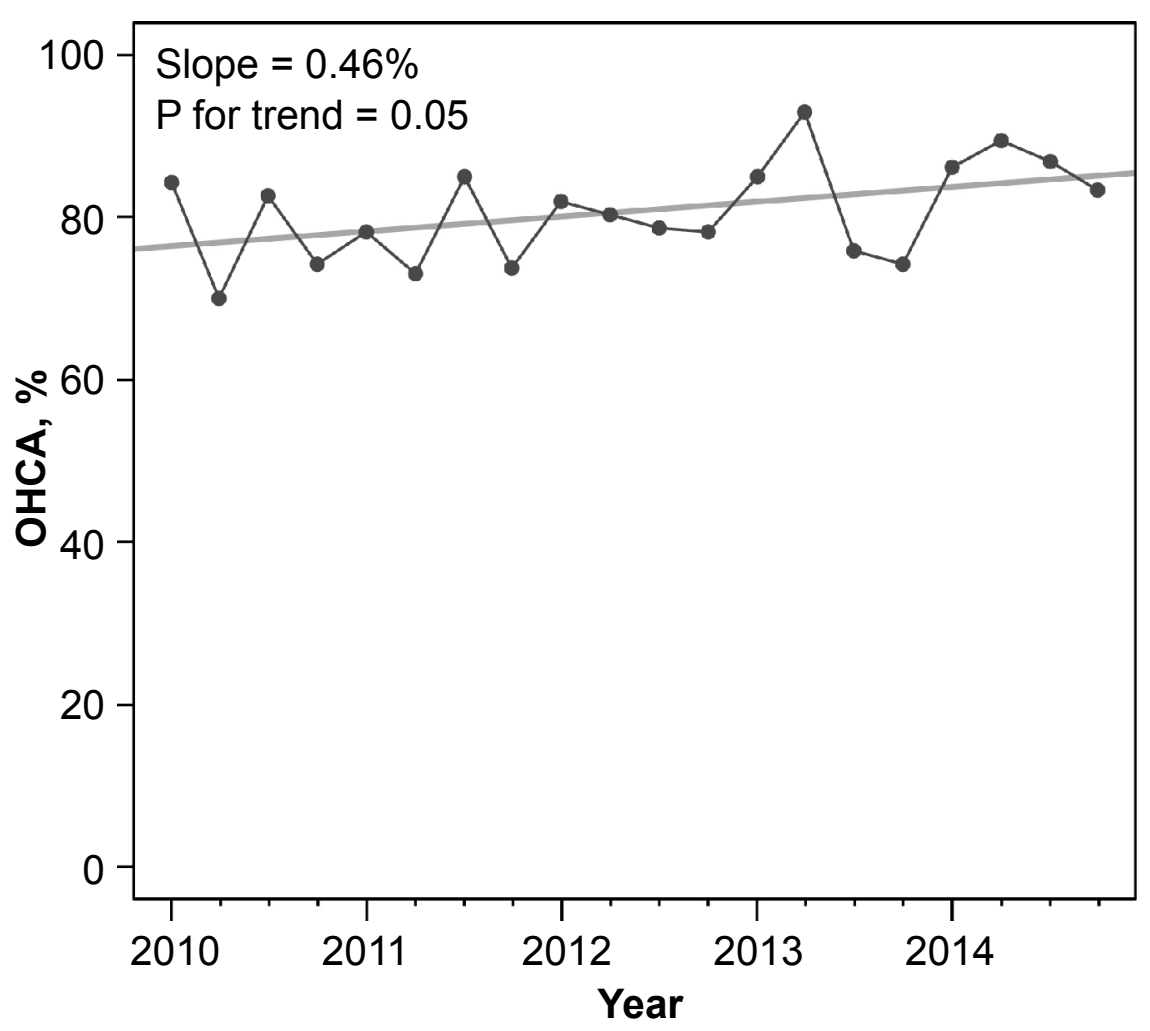

Figure 2: Trends in the proportion of TTM cases which were OHCA.

Over 20 calendar quarters between 2010 and 2014, there was a mild increase in the proportion of TTM cases with an OHCA.

Abbreviation: OHCA: Out-of-Hospital Cardiac Arrest. 
have a Shockable cardiac arrest rhythm than non-TTM patients.

Among the 976 patients treated with TTM, 398 (40.8\%) had a Shockable cardiac arrest rhythm of VF or VT whereas 523 (53.6\%) had a Non-Shockable cardiac arrest rhythm of asystole or PEA and 55 (5.6\%) had an Unknown cardiac arrest rhythm. Four in five TTM-treated patients (785/976 [80.4\%]) had an OHCA whereas $191(19.6 \%)$ had an IHCA. Table 2 summarizes several factors related to TTM treatment. The average duration of TTM treatment was $23.7 \pm 9.3$ hours, and the mean duration of re-warming was $13.4 \pm 6.6$ hours. Overall, 398 (40.8\%) of the TTM-treated patients survived to hospital discharge, and 309 (31.7\%) survived to discharge with favorable neurological status (i.e., a cerebral performance category score of 1 or 2 ).

Over 20 calendar year quarters, there was a mild

Table 2: Summary of TTM treatment and outcomes.

\section{TTM Variable}

Duration of TTM (hours \pm SD)

$23.7 \pm 9.3$

Duration Re-warming (hours $\pm \mathrm{SD}$ ) $13.4 \pm 6.6$

Clinical Outcomes

Survival to Hospital Discharge (\%)

Favorable Neurological Survival ${ }^{*}(\%)$

398 (40.8)

$309(31.7)$

${ }^{*}$ Favorable neurological survival was defined as survival to discharge with a cerebral performance category score of 1 or 2 , indicating no more than moderate neurological disability. trend ( $P$ for trend of 0.05 ) toward greater use of TTM in patients with OHCA (Figure 2), with the proportion gradually increasing from about $75 \%$ in the early quarters to over $80 \%$ in the later quarters. In contrast, the proportion of patients receiving TTM for a Non-Shockable cardiac arrest rhythm remained unchanged over time ( $P$ for trend of 0.35 ) (Figure 3). Lastly, among the 921 TTM-treated patients with a Known cardiac arrest rhythm, TTM was initiated for an OHCA due to a Shockable cardiac arrest rhythm-the setting in which TTM has been studied in randomized trials-in only $37.9 \%$ (349/921) of treated patients, althoughthis proportion has increased over time from $30.6 \%$ in the earlier quarters to over $45.2 \%$ in the later quarters ( $P$ for trend of 0.04) (Figure 4).

\section{Discussion}

In a contemporary multi-site registry of cardiac arrests treated with TTM, we found that TTM was instituted in $52 \%$ of patients during post-resuscitation care and that the majority were patients with OHCA. However, the majority of treated patients did not have a Shockable cardiac arrest rhythm of VF or VT, and only $38 \%$ of patients treated with TTM had an OHCA due to a Shockable rhythm. Moreover, although a greater proportion of TTM-treated patients have had an OHCA due to VF/VT over the 5-year study period this proportion of treated patients with documented efficacy by clinical trials

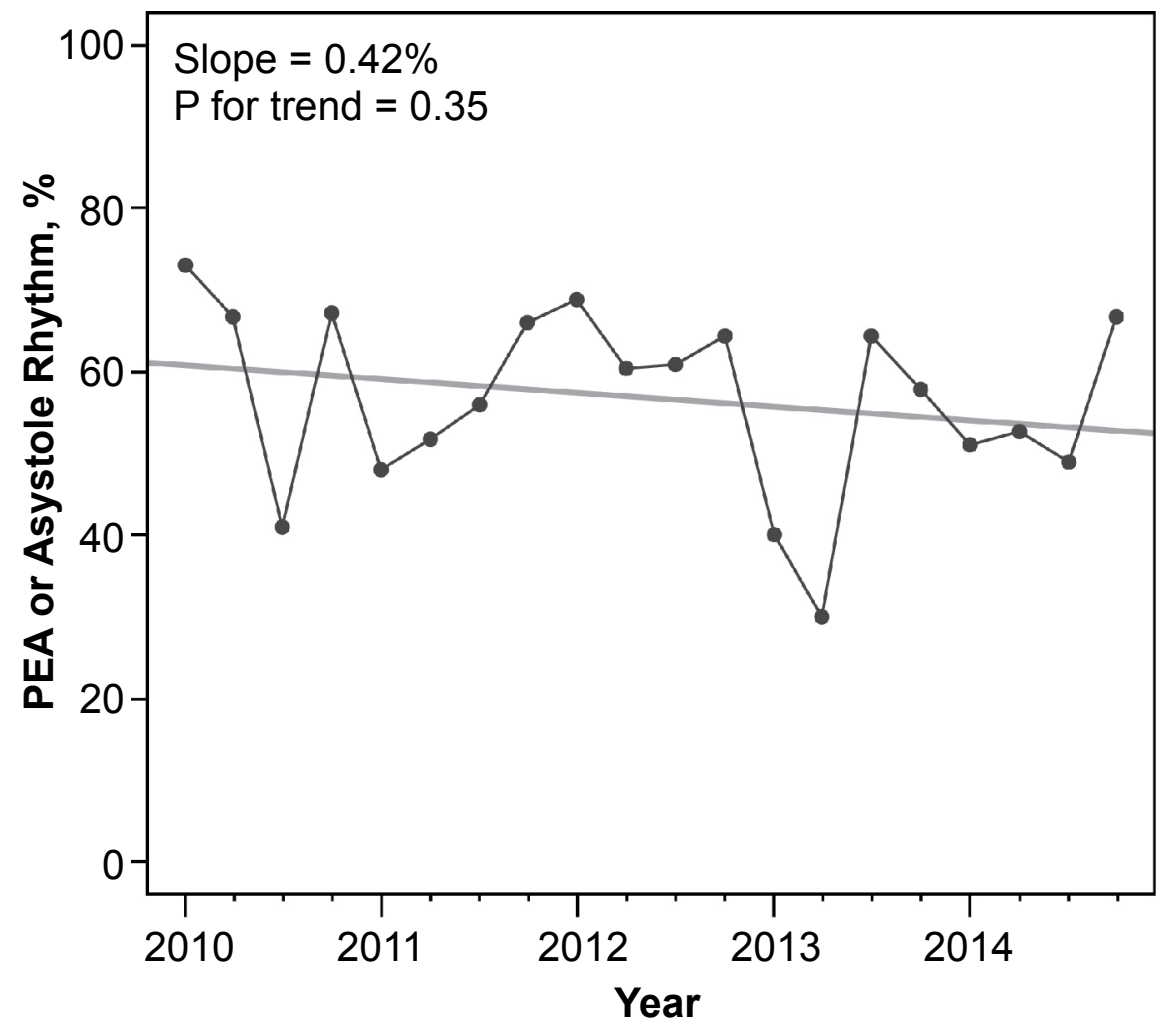

Figure 3: Trends in the proportion of TTM cases due to a non-shockable cardiac arrest rhythm.

Over 20 calendar quarters between 2010 and 2014, there was no change in the proportion of TTM cases for cardiac arrests due to non-shockable cardiac arrest rhythms (asystole or pulseless electrical activity), and non-shockable rhythms continued to comprise the majority of TTM cases for cardiac arrest.

Abbreviation: PEA: Pulseless Electrical Activity. 


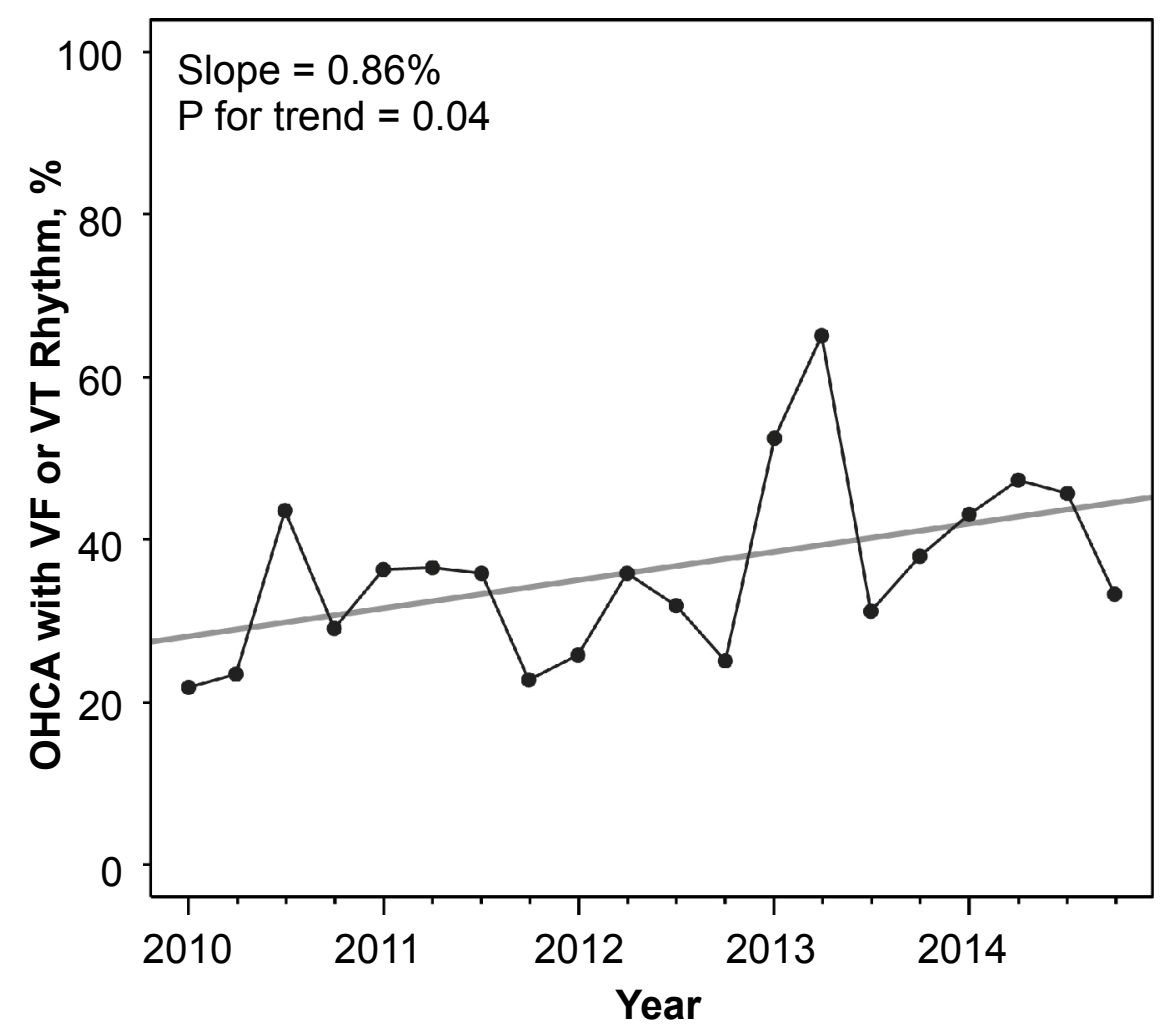

Figure 4: Trends in the proportion of TTM cases for OHCA due to a shockable cardiac arrest rhythm.

Over 20 calendar quarters between 2010 and 2014, there was a mild increase in the proportion of TTM cases with an OHCA due to VF or pulseless VT-the indication in which TTM has been shown to improve survival outcomes in randomized trials.

Abbreviations: OHCA: Out-of-Hospital Cardiac Arrest; VF: Ventricular Fibrillation; VT: Pulseless Ventricular Tachycardia.

remained less than half of all TTM-treated patients. Collectively, our findings provide important insights into which cardiac arrest patient populations are being treated with TTM in routine care.

The 2002 publication of two landmark clinical trials for TTM treatment ushered in a dynamic new treatment that is expected to have improved survival and neurological outcomes for patients with OHCA due to VF $[4,5]$. In routine practice, however, there appears to have been significant expansion of TTM treatment to other settings, such as Non-Shockable cardiac arrest rhythms and IHCA. This is underscored by recent guideline recommendations to consider use of TTM in these settings $[10,11]$. However, for IHCA, hospital response times in initiating cardiopulmonary resuscitation are likely more rapid than from bystanders during OHCA, the advantages of initiatingTTM treatment in the In-Hospital setting to reduce reperfusion injury due to prolonged brain anoxia may differ as compared with the Out-of-Hospital setting. To date, randomized trials of TTM for IHCA in adults do not exist but a recent trial of TTM in children for IHCA found no difference in In-Hospital and 1 year survival between those managed with TTM to 32 to 34 Celsius vs. a control group which focused on avoidance for fever [15]. Additionally, observational studies, though limited, have not found a consistent benefit of TTM for patients with IHCA. Kory, et al. found no difference in rates of survival to discharge between 16 non-
TTM patients (31\%) vs. 17 TTM-treated patients (24\%; P = NS) [7] and Nichol, et al. found no difference in rates of favorable neurological survival between 214 TTM-treated patients (18.7\%) vs. 8102 non-TTM patients (20.1\%), even after propensity score risk adjustment [8]. More recently, in the largest study, to date, of the association of TTM for IHCA, Chan, et al. found no survival benefit (and potentially harm) with TTM among 1524 treated patients compared with 3714 non-TTM-treated patients [9]. As a result, efficacy and comparative effectiveness data for the benefit of TTM treatment for IHCA remains lacking.

Randomized trials supporting TTM treatment in patients with Non-Shockable cardiac arrest rhythms of asystole or PEA have been similarly lacking. There have been a number of observational studies which have compared outcomes with TTM treatment among patients with Non-Shockable cardiac-arrest rhythms. Although earlier smaller studies reported improved outcomes, larger studies have not found that TTM treatment was associated with improved survival outcomes among patients with Non-Shockable cardiac arrest rhythms. For instance, Don, et al. found no difference in survival with TTMtreatment among patients with OHCA due to a Non-Shockable rhythm (odds ratio, 1.17, 95\% confidence interval, 0.66-2.05) [16]. A recent meta-analysis of 12 observational studies of TTM treatment in patients with Non-Shockable cardiac arrests found only a 
small non-significantdifference in rates of poor neurological outcome in a random-effects model (pooled OR, 0.95 [0.90-1.01]) [6]. Importantly, the authors assessed most studies were of low quality (e.g., small sample size and/or pre- vs. post-design with poor case-mix adjustment) and had significant potential for bias. Since that meta-analysis, a large study of 1145 OHCA patients found that TTM was associated with better neurological survival only in patients with Shockable rhythms but not in patients with Non-Shockable rhythms (OR, 0.71 [0.37-1.36]), while a different prospective study found no benefit with TTM treatment in 387 patients at hospital discharge (Hazard Ratio, 0.98 [0.53-1.50) or at 90-day follow-up ( $P=0.82)[17,18]$. Finally, Chan, et al. found that TTM was not beneficial and potentially harmful for patients with IHCA due to a Non-Shockable cardiac arrest rhythm [9].

In the absence of randomized trials, observational studies can sometimes provide insights to support a treatment's use outside the studied indication, especially since randomized trials are resource-intensive and may not always be practical. Prior observational studies of TTM, however, have not provided solid confirmation of benefit with TTM treatment for either IHCA or cardiac arrests due to Non-Shockable cardiac arrest rhythms. Current resuscitation guidelines do recommend TTM in all comatose survivors, but this is based on expert consensus. However, since TTM is not without cost or adverse effects, there is a critical need for prospective randomized trials to establish its clinical efficacy for asystole and PEA rhythms and IHCA, in light of the observational evidence and the one recent trial for pediatric IHCA. Since large national cardiac arrest registries for OHCA and IHCA already exist, it would be reasonable to leverage existing data collection infrastructure to conduct pragmatic trials of TTM. Given that more than 3 in 5 patients treated with TTM after cardiac arrest have indications not studied in randomized trials and without definitive support from observational studies, national registries provide a unique opportunity to prospectively study the clinical efficacy of TTM treatment for Non-Shockable cardiac arrest rhythms in both the In-Hospital and Out-of-Hospital setting.

Our study should be interpreted in the context of the following limitations. The PATH registry reflects contemporary TTM treatment for post-resuscitation care across 26 centers. Although these centers represent a diverse group of hospitals, patterns of TTM treatment may differ among hospitals not participating within the PATH registry. Second, we did not compare outcomes between patients treated and not treated with TTM. This is, in part, because we did not have an adequately large sample size to compare outcomes between treated and untreated patients and as comparative outcomes are outside the scope of this study. Nonetheless, even if we had a large study sample, it is unlikely that any observational study would be able to control for significant indication bias, which can only be addressed in the context of a randomized clinical trial.

In conclusion, we found that, although TTM continues to be used primarily in patients with OHCA, only 2 in 5 patients treated with TTM had an OHCA due to VF/VTthe indication for which TTM has been rigorously evaluated. Although the proportion of TTM-treated patients with this proven indication has modestly increased over the years, this indication still comprises less than half of all patients treated with TTM in contemporary practice. As the majority of cardiac arrest patients treated with TTM have either an IHCA or a Non-Shockable cardiac arrest rhythm for which the benefit of TTM treatment remains unclear, there remains a critical need to rigorously evaluate the clinical efficacy of TTM in these clinical settings. The existence of national registries may provide a unique opportunity to conduct pragmatic trials of TTM in these other cardiac arrest settings.

\section{Funding/Support}

Dr. Chan is supported by funding (K23HL102224 and R01HL123980) from the National Heart Lung and Blood Institute.

\section{Study Oversight}

Drs. Narsingam and Chan designed the study. Drs. Narsingam and Chan had full access to all of the data in the study, and take responsibility for the integrity of the data and the accuracy of the data analysis.

\section{References}

1. Mozaffarian D, Benjamin EJ, Go AS, Berry JD, Blaha MJ, et al. (2014) Heart Disease and Stroke Statistics-2015 Update A Report From the American Heart Association. Circulation 129: e28-e292.

2. Dohi K, Miyamoto K, Fukuda K, Nakamura S, Hayashi M, et al. (2013) Status of Systemic Oxidative Stress during Therapeutic Hypothermia in Patients with Post-Cardiac Arrest Syndrome. Oxid Med Cell Longev.

3. Lei B, Tan X, Cai H, Xu Q, Guo Q (1994) Effect of moderate hypothermia on lipid peroxidation in canine brain tissue after cardiac arrest and resuscitation. Stroke 25: 147-152.

4. Bernard SA, Gray TW, Buist MD, Jones BM, Silvester W, et al. (2002) Treatment of Comatose Survivors of Out-of-Hospital Cardiac Arrest with Induced Hypothermia. N Engl J Med 346: 557-563.

5. Hypothermia after Cardiac Arrest Study Group (2002) Mild Therapeutic Hypothermia to Improve the Neurologic Outcome after Cardiac Arrest. N Engl J Med 346: 549-556.

6. Kim YM, Yim HW, Jeong SH, Klem ML, Callaway CW (2012) Does therapeutic hypothermia benefit adult cardiac arrest patients presenting with non-shockable initial rhythms?: A systematic review and meta-analysis of randomized and non-randomized studies. Resuscitation 83: 188-196.

7. Kory P, Fukunaga M, Mathew JP, Singh B, Szainwald L, et al. (2012) Outcomes of mild therapeutic hypothermia after in-hospital cardiac arrest. Neurocrit Care 16: 406-412.

8. Nichol G, Huszti E, Kim F, Fly D, Parnia S, et al. (2013) 
Does induction of hypothermia improve outcomes after in-hospital cardiac arrest? Resuscitation 84: 620-625.

9. Chan PS, Berg RA, Tang Y, Curtis LH, Spertus JA, et al (2016) Association Between Therapeutic Hypothermia and Survival After In-Hospital Cardiac Arrest. JAMA 316: 13751382.

10. Callaway CW, Donnino MW, Fink EL, Geocadin RG, GoIan E, et al. (2015) Part 8: Post-Cardiac Arrest Care: 2015 American Heart Association Guidelines Update for Cardiopulmonary Resuscitation and Emergency Cardiovascular Care. Circulation 132: S465-S482.

11. Soar J, Callaway CW, Aibiki M, Böttiger BW, Brooks SC, et al. (2015) Part 4: Advanced life support: 2015 International Consensus on Cardiopulmonary Resuscitation and Emergency Cardiovascular Care Science with Treatment Recommendations. Resuscitation 95: e71-e120.

12. Leary M, Grossestreuer AV, lannacone S, Gonzalez M, Shofer FS, et al. (2013) Pyrexia and neurologic outcomes after therapeutic hypothermia for cardiac arrest. Resuscitation 84: 1056-1061.
13. Beylin ME, Perman SM, Abella BS, Leary M, Shofer FS, et al. (2013) Higher mean arterial pressure with or without vasoactive agents is associated with increased survival and better neurological outcomes in comatose survivors of cardiac arrest. Intensive Care Med 39: 1981-1988.

14. (2015) Penn Alliance for Therapeutic Hypothermia (PATH).

15. Moler FW, Silverstein FS, Holubkov R, Slomine B, Christensen J, et al. (2017) Therapeutic Hypothermia after In-Hospital Cardiac Arrest in Children. N Engl J Med 376: 318-329.

16. Don CW, Longstreth WT, Maynard C, Olsufka M, Nichol G, et al. (2009) Active surface cooling protocol to induce mild therapeutic hypothermia after out-of-hospital cardiac arrest: A retrospective before-and-after comparison in a single hospital. Crit Care Med 37: 3062-3069.

17. Dumas F, Grimaldi D, Zuber B, Fichet J, Charpentier J, et al. (2011) Is hypothermia after cardiac arrest effective in both shockable and nonshockable patients?: insights from a large registry. Circulation 123: 877-886.

18. Storm C, Nee J, Roser M, Jörres A, Hasper D (2012) Mild hypothermia treatment in patients resuscitated from nonshockable cardiac arrest. Emerg Med J 29: 100-103. 